“C 2017 IEEE. Personal use of this material is permitted. Permission from IEEE must be obtained for all other uses, in any current or future media, including reprinting/republishing this material for advertising or promotional purposes, creating new collective works, for resale or redistribution to servers or lists, or reuse of any copyrighted component of this work in other works." 


\title{
A Segmented Rotor Type Switched Reluctance Machine for BSGs of Hybrid Electric Vehicles: Concept, Design and Analysis
}

\author{
Zhuicai Zhou ${ }^{1}$, Xiaodong Sun ${ }^{1,2}$, Long Chen ${ }^{2}$, Zebin Yang ${ }^{3}$, Shouyi $\mathrm{Han}^{3}, \mathrm{Ke} \mathrm{Li}^{3}$, Jianguo Zhu ${ }^{4}$, and Youguang Guo ${ }^{4}$ \\ ${ }^{1}$ School of Automobile and Traffic Engineering, Jiangsu University, Zhenjiang 212013, China, xdsun@ ujs.edu.cn \\ ${ }^{2}$ Automotive Engineering Research Institute, Jiangsu University, Zhenjiang 212013, China \\ ${ }^{3}$ School of Electrical and Information Engineering, Jiangsu University, Zhenjiang 212013, China \\ ${ }^{4}$ School of Electrical, Mechanical and Mechatronic Systems, University of Technology, Sydney, Sydney, NSW 2007, Australia
}

\begin{abstract}
This paper proposes a novel 16/10 segmented rotor switched reluctance machine (SSRM) for belt-driven starter generators (BSGs) of hybrid electric vehicles. The stator of proposed SSRM consists of two types of stator poles: exciting pole and auxiliary pole, and the rotor of proposed SSRM is made up of a series of discrete segments. Firstly, the concept of the conventional SRM and proposed machine is presented. Secondly, the design rules of proposed SSRM are described. Finally, the finite element method (FEM) is employed to get the static characteristics of the proposed SSRM, including the magnetic flux distribution, magnetic flux density, inductance characteristic, torque characteristic and continuous torque. Result shows that the torque ripple of proposed SSRM is low.
\end{abstract}

Index Terms--Segmented rotor; exciting and auxiliary poles; discrete segments; static characteristics; finite element method, torque ripple.

\section{INTRODUCTION}

With the environmental pollution and oil shortage situation is getting worse, new energy vehicles began to be widely attention. In the new energy vehicles models, the development of hybrid electric vehicles is faster than other style models. The Belt-driven Starter Generator (BSG) is widely employed in hybrid electric vehicles. The induction machine is difficult to get the high torque at low speed [1], [2]. The permanent-magnet machine is prone to demagnetization under the high temperature [3]-[6]. Compared with the induction machines and permanentmagnet machines for BSGs of hybrid electric vehicles, switched reluctance machines (SRMs) have many advantages, such as low-cost, robustness, simple structure, etc. Due to these advantages, SRM has been widely concerned.

However, conventional SRMs also have some disadvantages, such as high torque ripple, low energy conversion density and low torque capability; these disadvantages limit the development of conventional SRM. To overcome these disadvantages, a novel 16/10 segmented rotor switched reluctance machines (SSRMs) for BSGs of hybrid electric vehicles is proposed in this paper.

The stator and segmental rotor of proposed SSRM is shown in Fig. 1. The novelty of the proposed machine lies in the use of auxiliary poles and the segmented rotor. The auxiliary poles are employed to produce the high fault- tolerance characteristic, and the segmented rotor can reduce torque ripples, hence improving the reliability of the proposed SSRM. The fault-tolerance ability of proposed machine is not discussed in this paper. The characteristics analysis is analyzed in [7]-[11].

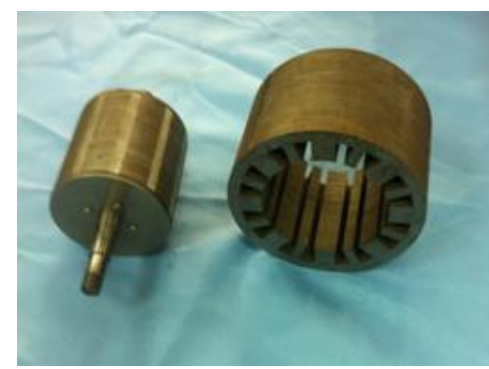

Fig. 1 Stator and segmental rotor of proposed machine.

In this paper, the concept of conventional SRM and proposed SSRM is described in section II. The design rule of proposed SSRM is introduced in section III. The static analysis of proposed SSRM is given in section IV. Finally, the conclusion is drowning in section $\mathrm{V}$.

\section{CONCEPT OF 16/10 SEgMENTAL Rotor TYPE SRM}

\section{A. Conventional 8/6 SRM}

The structure and winding connection type of conventional 8/6 SRM is shown in Fig. 2. From Fig. 2, it can be seen that phase A of conventional SRM is at the aligned position, and the connection type of winding is NSNSNSNS. In this situation, when phase B is excited, the rotor will rotate in the counterclockwise direction. In contrast, when phase D is excited, the rotor will rotate in clockwise direction.

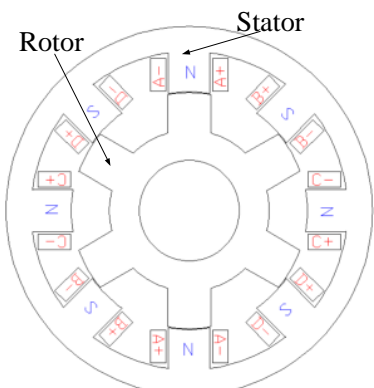

Fig. 2 Conventional 8/6 SRM.

Fig. 3 shows the magnetic flux path of phase A in conventional SRM. "+" represents the current flow out 
the paper, and "-" represents the current flow into the paper. It is found that the magnetic flux flows down the stator pole, flow through the air gap to the rotor pole and return via the adjacent stator pole.

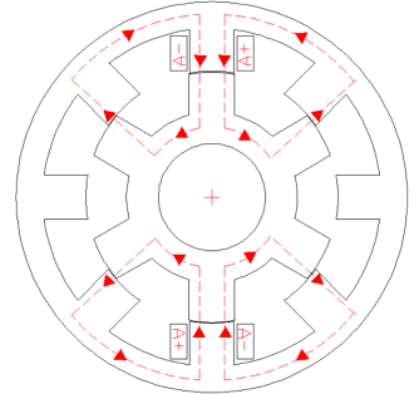

Fig. 3 Magnetic flux path of phase A in conventional SRM.

\section{B. Novel 16/10 Segmental Rotor Type SRM}

The structure and winding connection type of $16 / 10$ segmental rotor type SRM is introduced in Fig. 4. It is found that the stator of proposed SSRM comprises of two type of the stator poles: exciting and auxiliary poles. The auxiliary poles is not provided with a winding, and only provided the return path for the flux. In addition, the rotor of the proposed SSRM consists of a series of discrete segments, and all rotor segments are embedded in an aluminum rotor block. The new structure of proposed SSRM can greatly reduce the torque ripple, and increase the output torque. It can also found in Fig. 4 that the connection type of winding is NSNSNSNS. Assume that the position is in the phase $\mathrm{C}$ excited, when the order of excited is C-B-A-D, the rotor will rotate in the clockwise direction. In contrast, when the order of excited is C-D-A$\mathrm{B}$, the rotor will rotate in the counterclockwise direction.

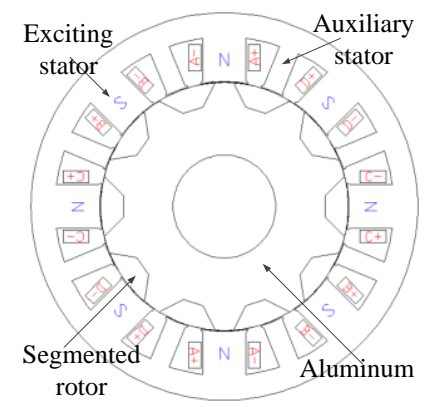

Fig. 4 16/10 segmental rotor type SRM.

The magnetic flux path of proposed SSRM with phase A energized is shown in Fig. 5. It is seen in Fig. 5 that the magnetic flux flows down the exciting stator, through a segmental rotor and then returns by the adjacent auxiliary stator.

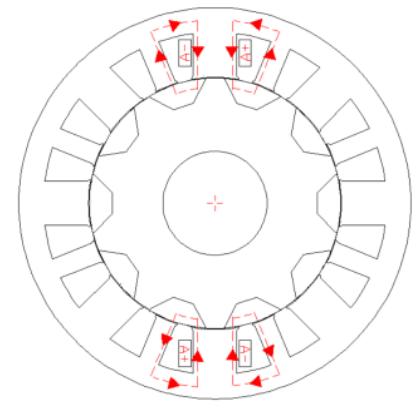

Fig. 5 Magnetic flux path of phase A in proposed SSRM.
Compared with the magnetic flux path of conventional SRM, the magnetic flux path of proposed SSRM is shorter. The short magnetic flux path is beneficial to reduce the core loss, and the non-salient rotor of proposed SSRM can reduce the wind or oil resistance.

\section{DESIGN OF 16/10 SEgMENTAL Rotor TYPE SRM}

In order to clarify the structure of proposed machine, a novel $16 / 10$ segmental rotor type SRM is proposed. The proposed SSRM is designed for BSG of hybrid electric vehicles $(60 \mathrm{~V}, 1.8 \mathrm{KW}$, and $6000 \mathrm{rpm})$. The following design rule is made to clarify the structure of proposed SSRM.

1. The pole arc coefficient of the excitation tooth is twice that of the auxiliary tooth, which is interpreted as the excitation stator carrying the flux is twice the auxiliary stator. The above relationship is given as:

$$
\alpha_{s 2}=2 \alpha_{s 1}
$$

where $\alpha_{s 2}$ is the pole arc coefficient of the exciting stator, and $\alpha_{s l}$ is the pole arc coefficient of the auxiliary stator.

2 . When the machine is in the position of the minimum inductance, in order to ensure that the magnetic reluctance is the largest, the distance between the adjacent rotor teeth is greater or equal to the distance between the adjacent stator teeth, which can be expressed as:

$$
\frac{2 \pi\left(1-\alpha_{r}\right)}{Z_{r}} \geq \frac{1}{2}\left(\frac{2 \pi}{8}-\frac{2 \pi \alpha_{s 1}}{Z_{s}}-\frac{2 \pi \alpha_{s 2}}{Z_{s}}\right)
$$

where $Z_{r}$ is the rotor pole number, $Z_{s}$ is the stator pole number, and $\alpha_{r}$ is the rotor pole arc coefficient.

3. The distance of rotor is greater to the distance of exciting stator, which can described as:

$$
\frac{2 \pi \alpha_{\mathrm{r}}}{Z_{r}}>\frac{2 \pi \alpha_{s 2}}{Z_{s}}
$$

According to (1)-(3), we can obtain the range of $\alpha_{r}$ and $\alpha_{s 2}$ which are illustrated in Fig. 6. That is, the area surrounded by OABC is the range of $\alpha_{r}$ and $\alpha_{s 2}$.

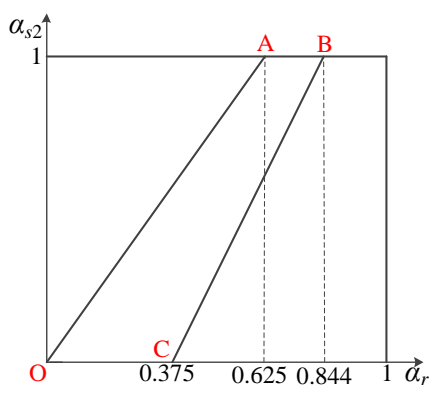

Fig. 6 Relationship between $\alpha_{r}$ and $\alpha_{s 2}$.

\section{ANALYSIS OF 16/10 SEGMENTAL ROTOR TYPE SRM}

The finite element method (FEM) is employed to get the characteristics of proposed SSRM. According to the process of design, we can obtain the optimization parameters as shown in Table I.

\section{A. Magnetic Flux Distribution}

The magnetic flux distributions of each conduction location are given in Fig. 7. When phase A, phase B, phase $C$ and phase $D$ at the aligned position is excited, the 
magnetic flux distributions is given in Figs. 7(a) - (d), respectively. It is found that the coupling between the adjacent slots is little, which means that the phases are working independent of each other. It is also found that the magnetic flux distribution verifies the correctness of the magnetic flux path. Beside, Fig. 7(e) introduces the magnetic flux distribution at the unaligned position. It is found that the magnetic flux leakage exists in the unaligned position.

TABLE I

THE PARAMETERS OF PROPOSED SSRM

\begin{tabular}{cc}
\hline \hline Parameters & Proposed SSRM \\
\hline Number of phase & 4 \\
Outer radius of stator(mm) & 64 \\
Yoke thickness of stator(mm) & 8 \\
Outer radius of rotor(mm) & 41 \\
Length of air gap $(\mathrm{mm})$ & 0.25 \\
Length of stack $(\mathrm{mm})$ & 80 \\
Stator pole $\operatorname{arc}\left({ }^{\circ}\right)$ & $21.375 / 10.69$ \\
Rotor pole $\operatorname{arc}\left({ }^{\circ}\right)$ & 26.64 \\
Number of turns & 26 \\
Wire diameter(mm) $(\mathrm{mm})$ & 2 \\
Resistance per phase $(\mathrm{m} \Omega)$ & 18.5 \\
Slot factor & 0.82 \\
Diameter of axle $(\mathrm{mm})$ & 17 \\
\hline
\end{tabular}

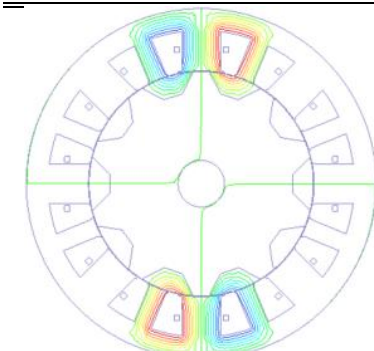

(a) Phase A

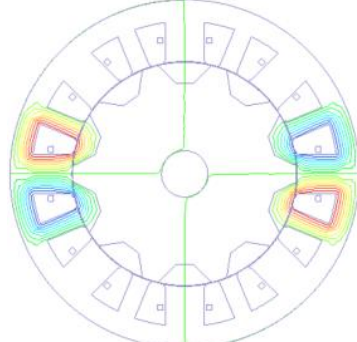

(c) Phase C

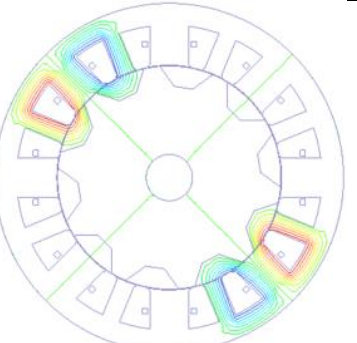

(b) Phase B

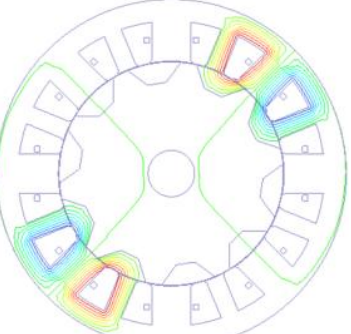

(d) Phase D.

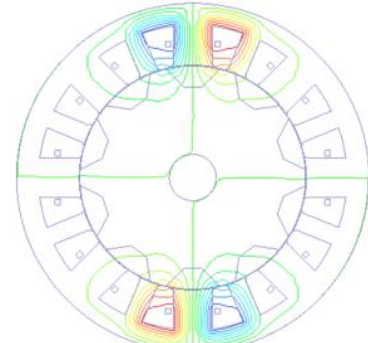

(e) Unaligned position.

Fig. 7 Magnetic flux distribution of proposed SSRM.

\section{B. Magnetic Flux Density}

The magnetic flux density of conventional SRM and proposed SSRM is given in Fig. 8. It is seen in Fig. 8 that the average magnetic flux density of conventional SRM and proposed SSRM in stator yoke is less than 1.48T, which meets the basis designed requirement of proposed SSRM.
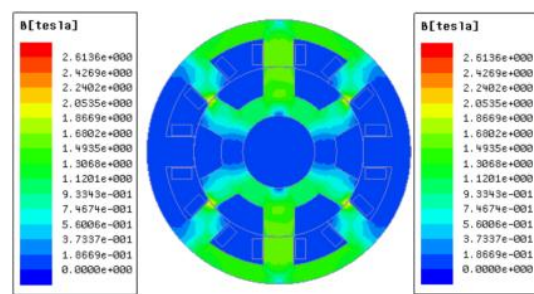

(a) Conventional $8 / 6 \mathrm{SRM}$

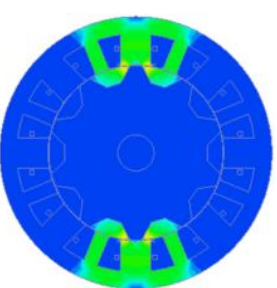

(b) Proposed SSRM
Fig. 8 Magnetic flux density of conventional SRM and proposed SSRM.

\section{Inductance and Torque Characteristics}

The inductance characteristic of proposed SSRM is given in Fig. 9. From Fig. 9, it can be seen that the inductance increases first and then decreases with the increases of rotor position angle, and starts to saturate when approaching the aligned position. That is because when the air gap flux increases to a certain value, the machine will reach a certain degree of saturation. It is also found that the inductance is substantially reduced with the increase of current level. The half mechanical cycle consists of a rotation between the unaligned position and the aligned position.

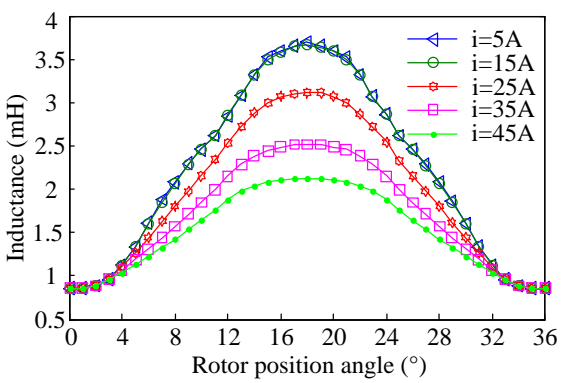

Fig. 9 Inductance profile.

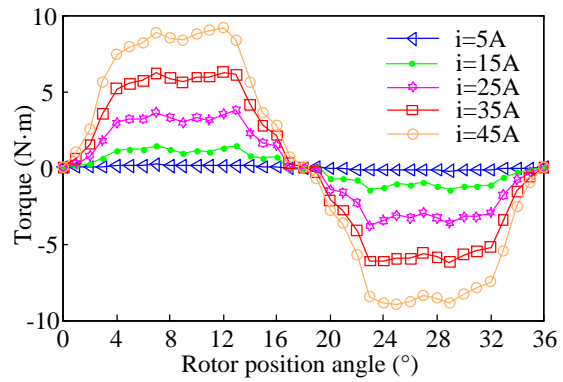

Fig. 10 Torque profile.

The torque is as described by:

$$
T=\frac{1}{2} i^{2} \frac{\partial L(i, \theta)}{\partial \theta}
$$

where $T$ is the torque, $i$ is the current, $\theta$ is the rotor position angle, and $L$ is the inductance.

The torque characteristic of proposed SSRM is introduced in Fig. 10. It is seen in Fig. 10 that the values of torque increases first and then decreases with the increases of rotor position angle under the half mechanical cycle, and the torque is substantially increases with the increase of current level. It is also seen in Fig. 10 that the torque is zero at rotor position angle $0^{\circ}, 18^{\circ}$ and $36^{\circ}$ since the flux is symmetry and the magnetic pull is zero. From Fig. 10, it can be seen that the torque starts to rise sharply when the rotor position angle is about $3^{\circ}$, which is related to the slope of the magnetic total energy change curve of the machine. 


\section{Continuous Torque}

The electromagnetic characteristics of every phase are considered for independent, thus the magnetic interactions is very small and can be ignored. In order to analysis the average torque of proposed SSRM, the continuous torque is shown in Fig. 10. It can be seen in Fig. 10 that the average torque is $2.865 \mathrm{~N} \cdot \mathrm{m}$. In order to realize the low torque ripple, it is desirable to have overlapping between two phases. From Fig. 10, it can be seen that two phases have overlapping parts, which means the torque ripple of proposed SSRM is relatively low.

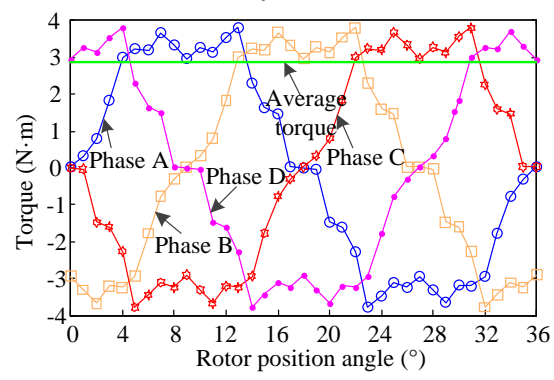

Fig. 10 Continuous torque.

\section{CONCLUSIONS}

A novel 16/10 segmented rotor SRM is designed in the paper. The paper has introduced the concept of conventional SRM and proposed SSRM. The winding connection type of two types SRM is NSNSNSNS. The paper verifies the magnetic flux path of conventional SRM is longer than that of proposed SSRM. The design of the stator and rotor pole arc coefficient is carried out. At last, the paper uses Ansys maxwell 2D for analysis. The magnetic flux distribution, magnetic flux density, inductance and torque characterizes is analyzed in this paper. In addition, the continuous torque of proposed SSRM is given to analysis the torque ripple. Result shows that the torque ripple of proposed SSRM is relatively low. In the further, some optimization method and strategies will be introduced [12]-[15].

\section{ACKNOWLEDGMENT}

This work was supported by the National Natural Science Foundation of China (51305170 and 51475214), the Natural Science Foundation of Jiangsu Province of China (BK20170071 and BK20141301), the Key Project of Natural Science Foundation of Jiangsu Higher Education Institutions (17KJA460005), the China Postdoctoral Science Foundation (2016M601726 and 2015T80508), the Six Categories Talent Peak of Jiangsu Province (2015-XNYQC-003, 2014-ZBZZ-017, and 2016-GDZB-096), Zhenjiang Key Research and Development Project (GY2016003), the "333 project" of Jiangsu Province (BRA2017441), and the Priority Academic Program Development of Jiangsu Higher Education Institutions (PAPD).

\section{REFERENCES}

[1] S. Chen, B. Lequesne, R. R. Henry, Y. Xue, and J. J. Ronning, "Design and testing of a belt-driven induction starter-generator", IEEE Trans. Ind. Appl., vol. 38, no. 6, pp. 1525-1533, Dec. 2002.

[2] X. Sun, L. Chen, Z. Yang, and H. Zhu, "Speed-sensorless vector control of a bearingless induction motor with artificial neural network inverse speed observer", IEEE/ASME Trans. Mech., vol. 18, no. 4, pp. 1357-1366, Aug. 2013.

[3] X. Sun, L. Chen, and Z. Yang, "Overview of bearingless permanent magnet synchronous motors", IEEE Trans. Ind. Electron., vol. 60, no. 12, pp. 5528-5538, Dec. 2013.

[4] X. Sun, L. Chen, H. Jiang, Z. Yang, J. Chen, and W. Zhang, "High-performance control for a bearingless permanent magnet synchronous motor using neural network inverse scheme plus internal model controllers", IEEE Trans. Ind. Electron., vol. 63, no. 6, pp. 3479-3488, June. 2016.

[5] X. Sun, Z. Shi, L. Chen, and Z. Yang, "Internal model control for a bearingless permanent magnet synchronous motor based on inverse system method", IEEE Trans. Energy Convers., vol. 31, no. 4, pp. 1539-1548, Dec. 2016.

[6] X. Sun, B. Su, L. Chen, Z. Yang, X. Xu, and Z. Shi, "Precise control of a four degree-of-freedom permanent magnet biased active magnetic bearing system in a magnetically suspended direct-driven spindle using neural network inverse scheme", in Proc. Mechanical Systems and Signal Processing (MSSP 2017), vol. 88, pp. 36-48, May. 2017.

[7] H. Zhang, D. H. Lee, and J. W. Ahn, “A segmental rotor type 12/8 switched reluctance motor: Concept, design and analysis", in Proc. 15th Int. Conf. IEEE ICIT, Feb. 2014

[8] J. Oyama, T. Higuchi, T. Abe, and K. Tanaka, "The fundamental characteristics of novel switched reluctance motor with segment core embedded in aluminum rotor block", J. Elect. Eng. Technol., vol. 1, no. 1, pp. 58-62, Oct. 2006.

[9] H. Zhang, D. H. Lee, C. W. Lee, and J. W. Ahn, "Design and analysis of a segmental rotor type 12/8 switched reluctance motor", J. Power Electron., vol. 14, no. 5, pp. 866-873, Sep. 2014.

[10] B, Wang, and J. W. Ahn, "Design and characteristic analysis of a novel axial field SRM with single teeth stator and segmental rotor", in Proc. International Conference on Electrical Machines and Systems (ICEMS 2013), pp. 571576, Oct. 2013.

[11] N. Vattikuti, V. Rallabandi, and B. G. Fernandes, "A novel high torque and low weight segmented switched reluctance motor", in Proc. Power Electronics Specialists Conference (PESC 2008), pp. 1223-1228, July. 2008.

[12] G. Lei, Y. G. Guo, J. G. Zhu, X. M. Chen, and W. Xu, "Sequential subspace optimization method for electromagnetic devices design with orthogonal design technique", IEEE Trans. Magn., vol. 48, no. 2, pp. 479-482, Feb. 2012.

[13] G. Lei, G. Y. Yang, K. R. Shao, Y. G. Guo, J. G. Zhu, and J. D. Lavers, "Electromagnetic device design based on RBF models and two new sequential optimization strategies", IEEE Trans. Magn., vol. 46, no. 8, pp. 3181-3184, Sep. 2010.

[14] G. Lei, C. C. Liu, J. G. Zhu, and Y. G. Guo, "Multilevel robust design optimization of a superconducting magnetic energy storage based on a benchmark study", IEEE Trans. Appl. Supercon., vol. 26, no. 7, pp. 1-5, Oct. 2016.

[15] G. Lei, K. R. Shao, Y. G. Guo, J. G. Zhu, and J. D. Lavers, "Improved sequential optimization method for high dimensional electromagnetic optimization problems", IEEE Trans. Magn., vol. 45, no. 10, pp. 3993-3996, Nov. 2009. 